Vibration absorption using non-dissipative complex attachments with impacts and parametric stiffness

N. Roveri, A. Carcaterra, and A. Akay

Citation: The Journal of the Acoustical Society of America 126, 2306 (2009); doi: 10.1121/1.3212942

View online: http://dx.doi.org/10.1121/1.3212942

View Table of Contents: http://asa.scitation.org/toc/jas/126/5

Published by the Acoustical Society of America 


\title{
Vibration absorption using non-dissipative complex attachments with impacts and parametric stiffness
}

\author{
N. Roveri \\ Department of Mechanics and Aeronautics, University of Rome, "La Sapienza," Via Eudossiana, 18, \\ 00184 Rome, Italy

\begin{abstract}
A. Carcaterra ${ }^{a)}$
Department of Mechanics and Aeronautics, University of Rome, "La Sapienza," Via Eudossiana, 18, 00184 Rome, Italy and Department of Mechanical Engineering, Carnegie Mellon University, Pittsburgh, Pennsylvania 15213
\end{abstract}

\author{
A. Akay \\ Department of Mechanical Engineering, Bilkent University, Ankara 06800, Turkey and Department of \\ Mechanical Engineering, Carnegie Mellon University, Pittsburgh, Pennsylvania 15213
}

(Received 31 October 2008; revised 30 July 2009; accepted 31 July 2009)

\begin{abstract}
Studies on prototypical systems that consist of a set of complex attachments, coupled to a primary structure characterized by a single degree of freedom system, have shown that vibratory energy can be transported away from the primary through use of complex undamped resonators. Properties and use of these subsystems as by energy absorbers have also been proposed, particularly using attachments that consist of a large set of resonators. These ideas have been originally developed for linear systems and they provided insight into energy sharing phenomenon in large structures like ships, airplanes, and cars, where interior substructures interact with a master structure, e.g., the hull, the fuselage, or the car body. This paper examines the effects of nonlinearities that develop in the attachments, making them even more complex. Specifically, two different nonlinearities are considered: (1) Those generated by impacts that develop among the attached resonators, and (2) parametric effects produced by time-varying stiffness of the resonators. Both the impacts and the parametric effects improve the results obtained using linear oscillators in terms of inhibiting transported energy from returning to the primary structure. The results are indeed comparable with those obtained using linear oscillators but with special frequency distributions, as in the findings of some recent papers by the same authors. Numerically obtained results show how energy is confined among the attached oscillators. (C) 2009 Acoustical Society of America. [DOI: 10.1121/1.3212942]
\end{abstract}

PACS number(s): 43.40.At, 43.40.Kd, 43.40.Jc, 43.40.Tm [ADP] Pages: 2306-2314

\section{INTRODUCTION}

An extensive literature exists on energy distribution in prototypical systems that consist of a set of linear parallel undamped resonators, called here as the attachment, all connected to a common vibrating structure, and often referred to as the primary or master structure. The pioneering work of Pierce et al. ${ }^{1}$ investigated a plate with a complex attachment demonstrating its unconventional damping property in the frequency-domain, and in Refs. 2 and 3 the problem is reconsidered, looking at the properties of a prototype master structure with attached set of weakly damped resonators. In Ref. 4, the damping effect produced by this prototypical system is analytically demonstrated, even independently of any local energy dissipation, for an infinite number of resonators and with a particular frequency distribution. The problem was further analyzed, focusing on the temporary nature of the energy storage for a finite number of attached resonators ${ }^{5}$ and on the energy redistribution process and equipartition in large undamped resonators. ${ }^{6}$ In Ref. 7 the intrinsic properties

\footnotetext{
a) Author to whom correspondence should be addressed. Electronic mail: a.carcaterra@dma.ing.uniroma1.it
}

of attachments are identified, which control the speed of energy sharing between a master and the attachment and the time the energy takes to be transferred back to the master. Several studies examined the conditions that, even in the absence of energy dissipation, prevent energy transport back to the master, which lead to the so called near-irreversibility condition $^{8-10}$ also confirmed by experimental tests. ${ }^{11}$ Finally, the problem of an efficient design of a multi-degrees of freedom tuned-mass-damper has been also considered in the context of control theory. ${ }^{12}$

In all these studies, energy redistribution process is considered in the framework of (i) linear interaction between the master and the attached resonators and (ii) in the absence of any direct interaction among the resonators, except through their reactions on the primary.

This paper addresses the effects of nonlinearities on energy transport by introducing nonlinear interaction-elastic collisions - among the resonators and a parametric instantaneous variation in the stiffness of the attached oscillators. The motivation for investigating these effects is summarized briefly as follows.

(a) In Ref. 10 it is shown how a damping effect on the 
master develops due to the attachment only when the uncoupled natural frequency of the master belongs to the interval $B$ described by the natural frequencies of the oscillators within the attachment. Conversely, if the master frequency falls outside of this bandwidth $B$, the energy sharing process is inhibited, significantly decoupling the master and the attachment.

(b) Under the conditions of the first point in case (a), most of the energy is transferred form the master to a limited number of resonators, i.e., to those oscillators having their natural frequencies closer to that of the master, thus concentrating the energy over a limited part of the attachment. $^{7}$

(c) Energy is continuously transferred and stored into the attachment for a period of time, but after a characteristic return time, ${ }^{7}$ it is transferred back to the master.

These observations naturally lead to investigating means to produce permanent energy storage within the attachment by modification of the linear system.

The behavior described in case (a) suggests that the master and the attachment can be energy-coupled or decoupled by just modifying the characteristic frequency distribution within the attachment during the vibration process as follows. In a linear system initially with a frequency distribution tuned with the master frequency, the energy is transferred from the master to the attachment. Following this transfer, when the condition of energy flow inversion from the attachment to the master becomes imminent (and this condition can be even theoretically predicted as in Ref. 7), the frequencies of the resonators of the attachment are suddenly modified, moving them far away from the master frequency, creating an energy-decoupling condition, and "freezing" the energy within the attachment. This strategy described in Sec. III.

An alternative approach, which amounts to producing an energy spreading effect, is to introduce direct interactions among the resonators within the attachment, permitting to them to have free and direct energy exchange. As described in Sec. II, letting oscillators develop impacts among them redistributes energy from those most energized to the others.

These nonlinear techniques also produce a nearirreversible energy transfer between the master and the attachment similar to that described in Ref. 10 for linear systems but using special frequency distribution within the attachment.

\section{IMPACTS WITHIN THE ATTACHMENT}

The prototypical two degrees of freedom system that produces impacts between adjacent oscillators, is schematically described in Fig. 1, with $m, k_{1}, k_{2}, x_{1}(t)$, and $x_{2}(t)$ representing mass, stiffness $\left(k_{1}, k_{2}\right)$, and displacement of each resonator, respectively. It represents the characteristic module for elastic collision interaction used in the more general attachment investigated ahead, involving indeed multiple resonators, and its preliminary analysis helps in a better understanding of the general case.

The nonlinear behavior emerges as the relative distance $\left|x_{1}(t)-x_{2}(t)\right|$ equals the gap $g$ and an impact between the

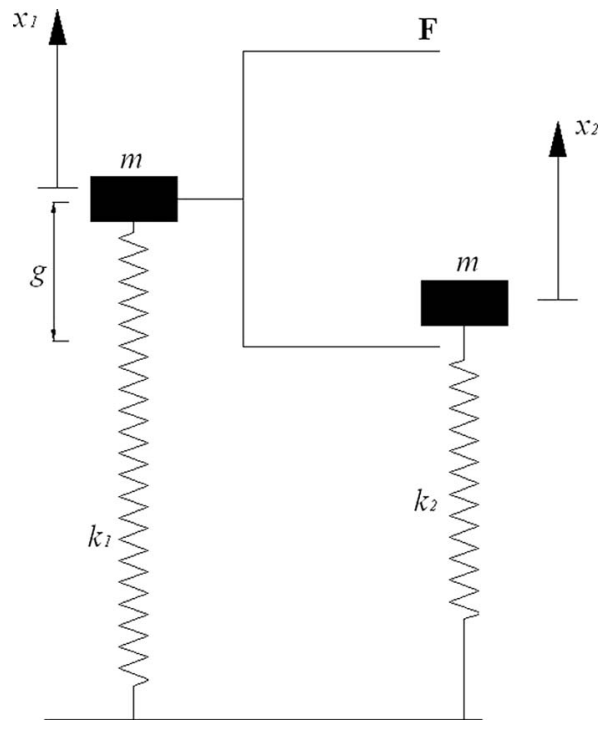

FIG. 1. The two-resonator impact-coupling.

resonators takes place through the impact frame F. An assumption of perfect elastic collision is made. The equations of energy and the momentum conservation imply

$$
\begin{aligned}
& \left(\dot{x}_{1}^{2}\left(t_{+}\right)+\dot{x}_{2}^{2}\left(t_{+}\right)\right) \frac{m}{2}=\left(\dot{x}_{1}^{2}\left(t_{-}\right)+\dot{x}_{2}^{2}\left(t_{-}\right)\right) \frac{m}{2}, \\
& m\left(\dot{x}_{1}\left(t_{+}\right)-\dot{x}_{1}\left(t_{-}\right)\right)=-m\left(\dot{x}_{2}\left(t_{+}\right)-\dot{x}_{2}\left(t_{-}\right)\right),
\end{aligned}
$$

where $t_{-}$and $t_{+}$are the time just preceding and subsequent to the impact, respectively. It follows

$$
\begin{aligned}
& \dot{x}_{1}\left(t_{+}\right)=\dot{x}_{2}\left(t_{-}\right), \\
& \dot{x}_{2}\left(t_{+}\right)=\dot{x}_{1}\left(t_{-}\right),
\end{aligned}
$$

meaning the resonators just exchange their velocities during an impact. Equation (2) is used to study the impacts within the complete attachment consisting of a plurality of resonators.

Therefore, the complete system represented in Fig. 2, in the absence of external forces, is described by the equations

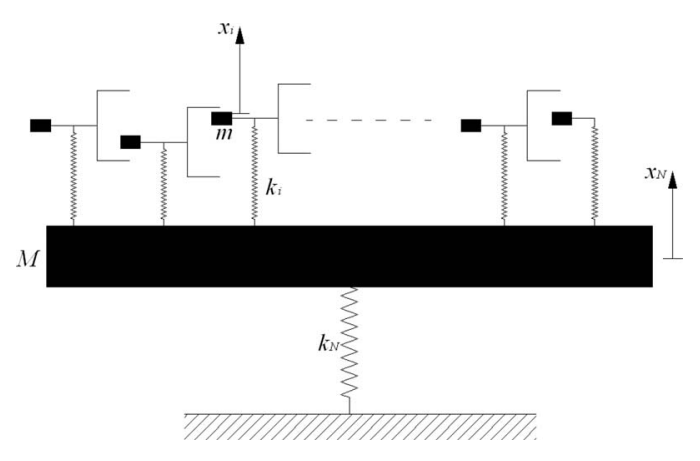

FIG. 2. Master-attachment prototype system. 


$$
\begin{gathered}
m \ddot{x}_{j}(t)+k_{j}\left(x_{j}(t)-x_{N}(t)\right)=\sum_{k} I_{k}^{j, i} \delta\left(t-t_{k}\right), \\
j=1,2, \ldots, N-1, \\
M \ddot{x}_{N}(t)+k_{N} x_{N}(t)+\sum_{j=1}^{N-1} k_{j}\left(x_{N}(t)-x_{j}(t)\right)=0,
\end{gathered}
$$

where the index $N$ designates the master, $1,2, \ldots, N-1$ are used for the oscillators of the attachment, $m, k_{j}, M, k_{N}, x_{j}(t)$, and $t$ are the mass and the stiffness of each oscillator of the attachment, the mass and the stiffness of the master, the displacement of the $j$ th oscillator, and time, respectively, $I_{k}^{j, i}$ represents the impulse exchanged between the $j$ th and the $i$ th resonators at time $t_{k}, I_{k}^{i, j}=-I_{k}^{j, i}$, and $\delta\left(t-t_{k}\right)$ is the Dirac delta function. However, accordingly with the system depicted in Fig. 2, the elastic collision interactions represented by $I_{k}^{j, i}$ are restricted to the resonators with the nearest neighbors.

Matrix form for Eq. (3) reads

$$
\mathbf{M} \ddot{x}+\mathbf{K} x=f(x, \dot{x}),
$$

where $\mathbf{M}$ and $\mathbf{K}$ are the mass and stiffness matrices, and $f(x, \dot{x})$ represents the conservative, internal, and impact forces.

Equation (4) is piecewise linear and an iterative analytic solution at each iteration step can be expressed as

$$
\begin{aligned}
x\left(x_{0}, \dot{x}_{0}, t, t_{0}\right)= & \sum_{r=1}^{N}\left[\mathbf{u}_{r}^{T} \mathbf{M} x_{0} \cos \left(\omega_{r} t-\omega_{r} t_{0}\right)\right. \\
& \left.+\mathbf{u}_{r}{ }^{T} \mathbf{M} \dot{x}_{0} \frac{1}{\omega_{r}} \sin \left(\omega_{r} t-\omega_{r} t_{0}\right)\right] \cdot \mathbf{u}_{r},
\end{aligned}
$$

where $\omega_{r}$ and $\mathbf{u}_{r}$ are the eigenfrequency and the corresponding eigenvector, respectively, $x_{0}$ and $\dot{x}_{0}$ represent the initial displacement and velocity at $t_{0}$, respectively. Expression (5) is used iteratively to build the solution $s^{k}(t)$, which is a set of continuous functions for each time interval $\left[t_{k}, t_{k+1}\right)$, within which no impact takes place. For $t \in\left[0, t_{1}\right)$ Eq. (5) yields

$$
s^{0}(t)=x\left(x_{0}, \dot{x}_{0}, t, t_{0}\right) \quad \forall t \in\left[0, t_{1}\right) .
$$

With the initial conditions at $t_{0}=0$,

$$
x_{0}=\left\{\begin{array}{c}
0 \\
\vdots \\
0
\end{array}\right\} ; \quad \dot{x}_{0}=\left\{\begin{array}{c}
0 \\
\vdots \\
V_{0}
\end{array}\right\} \text {. }
$$

For $t \in\left[t_{1}, t_{2}\right)$ Eq. (5) becomes

$$
s^{1}(t)=x\left(x_{0}, \dot{x}_{0}, t, t_{0}\right) \quad \forall t \in\left[t_{1}, t_{2}\right)
$$

with initial condition on displacement as

$$
x_{0}=s^{0}\left(t_{1^{-}}\right) \text {. }
$$

The initial velocities are obtained using Eq. (2) for each impacting pair of resonators $j$ and $i$ at $t_{1}$

$$
\begin{aligned}
& \dot{x}_{0 j}=\dot{s}_{i}^{0}\left(t_{1-1}\right) \\
& \dot{x}_{0 i}=\dot{s}_{j}^{0}\left(t_{1-1}\right)
\end{aligned}
$$

Finally, for each oscillator $h$ that does not undergo an impact,

$$
\dot{x}_{0 h}=\dot{s}_{h}^{0}\left(t_{1^{-}}\right)
$$

is the initial condition at $t_{0}=t_{1}$.

The computational process starts with Eqs. (6)-(11), iteratively repeated up to the desired end time.

It would be emphasized how this procedure leads to a piecewise continuous solution using linear analysis within time spans between impacts in conjunction with velocity rules, given by Eq. (2), which impose velocity discontinuities on the resonators.

The model represented by Eqs. (1)-(11) is used to describe the energy sharing process between the master and the attachment. In Sec. IV the energy time history of the master $E_{N}(t)=1 / 2 M\left(\dot{x}_{N}^{2}+\omega_{M}^{2} x_{N}^{2}\right)$ and its time average $\lim _{T \rightarrow \infty} 1 / T \int_{0}^{T} E_{N}(t) d t$, where $\omega_{M}=\sqrt{k_{N} / M}$, are considered together with the average energy of the satellite oscillators.

\section{PARAMETRIC EFFECTS: TIME-VARYING STIFFNESS}

Several previous studies of the linear oscillators have shown how initially imparted energy to a master migrates to the attached oscillators. ${ }^{2,6,7}$ In particular, these studies have also shown how special frequency distributions of the oscillators enhance the transport of energy rapidly from the master to the oscillators. ${ }^{7}$ Theoretical, numerical, as well as experimental evidences of this phenomenon have been offered in Refs. 7-10. These results show that energy exchange between the master and its satellites takes place through a preferential frequency bandwidth $B$, as pointed out in case (a) in Sec. I, which must contain the master frequency, while the energy sharing process is inhibited when the master frequency falls outside this bandwidth.

Based on these considerations, the concept proposed here employs parametrically variable stiffness, with instantaneous variations, for the satellite oscillators; after an initial tuning period during which the master frequency falls within $B$, the satellite frequencies are shifted in a way that the master frequency is left outside $B$. Thus, the energy sharing process is inhibited before the energy can return to the master, confining the energy permanently within the attachment. Such a system, analogous to the one considered in Sec. II, still behaves linearly in each time interval.

The satellite oscillators all have equal mass $m$, while their initial stiffness is selected within the set $S \equiv\left\{k_{r}, r\right.$ $=1, \ldots, N-1 \mid k_{r} \neq k_{s}$ for $\left.r \neq s\right\}$. The initial value of the timevarying stiffness $\chi_{i}(t)$ of the $i$ th oscillator falls within $S$.

Of the two approaches proposed here to parametrically vary stiffness, the simpler one uses a time-dependent stiffness $\chi_{i}(t)$ defined as

$$
\begin{aligned}
& \chi_{i}(t)=k_{i}+\Delta k_{i} H\left(t-t^{*}\right), \quad \text { and } \quad k_{i} \in S, \\
& \sqrt{\frac{k_{i}+\Delta k_{i}}{m}}>\sqrt{\frac{k^{\max }}{m}}, \quad \forall i,
\end{aligned}
$$

where $H$ is the Heaviside step function and $k^{\max }=\max \left\{k_{i}\right\}$, $\Delta k_{i}>0$.

Roveri et al.: Attachments with impacts and parametric stiffness 


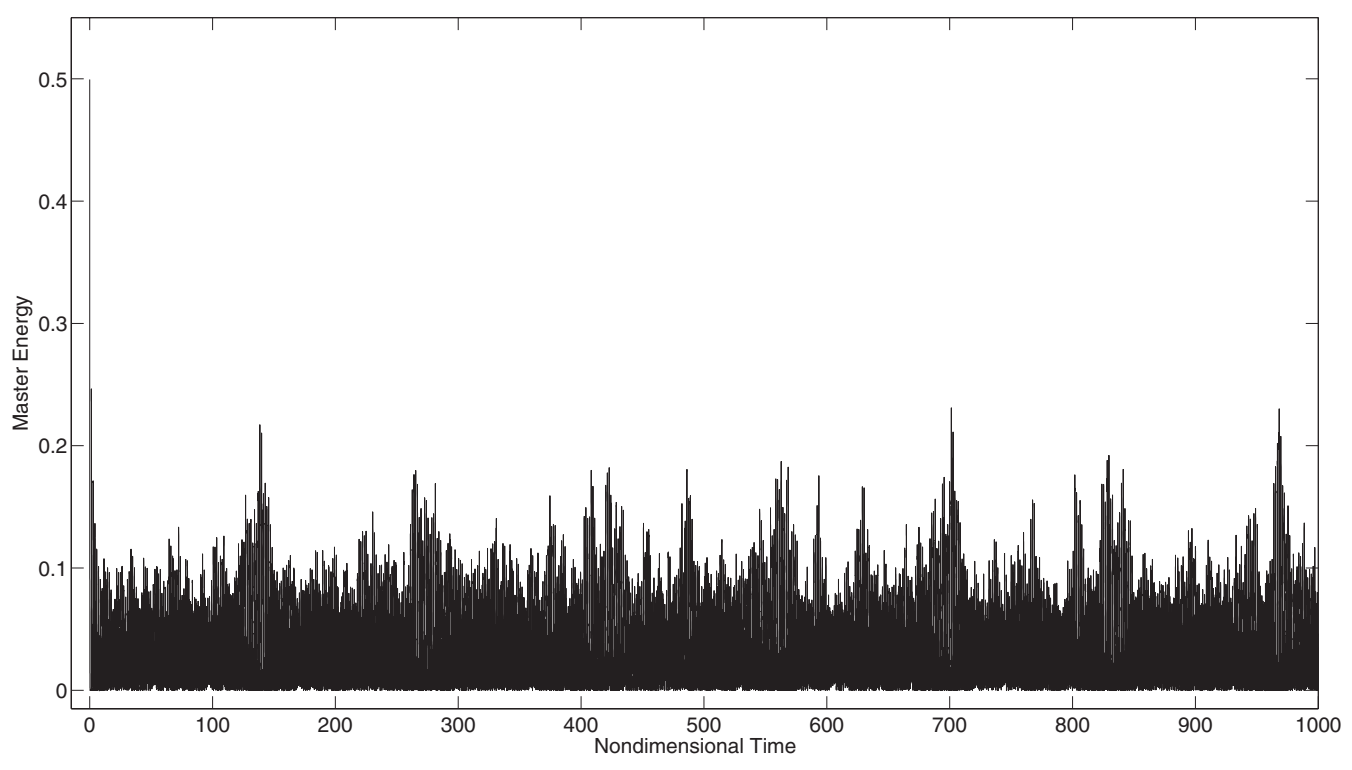

FIG. 3. Energy time history of the master for the linear system.

The initial uncoupled oscillator frequencies $\omega_{r}=\sqrt{k_{r} / m}$ all belong to the bandwidth $B \equiv\left\{0, \sqrt{k^{\max } / m}\right\}$, which includes the master frequency $\omega_{M}$.

The stiffness $\chi_{i}$ takes values, after a period $t^{*}$, within a set $T \equiv\left\{k_{r}+\Delta k_{r}, r=1, \ldots, N-1\right\}$, defined in Eq. (13). Equation (13) implies that for $t \geq t^{*}$, the all oscillator frequencies moved away from the bandwidth $B$, thus inhibiting energy sharing between the master and the satellite oscillators beyond time $t^{*}$, freezing the energy within the attachment.

Note in this case how, without prescribed values for $\Delta k_{i}$, except as described in Eq. (13), the frequency distribution $\sqrt{k_{i}+\Delta k_{i} / m}$ obtained $t>t^{*}$ differs from the initial one $\sqrt{k_{i} / m}$, and that the stiffness values $\chi_{i}$ for $t>t^{*}$ no longer belong to $S$, i.e., $S$ and $T$ have an empty intersection.

The second strategy for the parametric stiffness control uses the same frequency distribution at all times $t$, i.e., the stiffness of the attachment always belongs to the same set $S$ at all times. This second procedure follows the steps described below.

(1) The oscillators within the attachment are subdivided into two groups: $R^{(L)}$ and $R^{(H)}$. Those included in $R^{(H)}$ retain most of the total energy (as shown in Sec. IV), the remaining belong to $R^{(L)}$. In general, the number $N_{L}$ of resonators of $R^{(L)}$ exceeds the number $N_{H}$ of resonators of $R^{(H)}$.

(2) After time $t^{*}$, the stiffnesses $\chi_{r}^{(H)}(t)\left(r=1, \ldots, N_{H}\right)$ of the resonators in the group $R^{(H)}$, are simply interchanged with some of the stiffness $\chi_{i}^{(L)}(t)\left(i=1, \ldots, N_{L}\right)$ belonging to group $R^{(L)}$. The following expressions express this process formally:

$$
\begin{aligned}
& \chi_{r}^{(H)}(t)=k_{r}+\left[\chi_{s}^{(L)}(t)-k_{r}\right] H\left(t-t^{*}\right), \\
& r=1,2, \ldots, N_{H}, \quad s \in\left\{1,2, \ldots, N_{L}\right\}, \\
& \chi_{s}^{(L)}(t)=k_{s}+\left[\chi_{s}^{(H)}(t)-k_{s}\right] H\left(t-t^{*}\right) .
\end{aligned}
$$

Because of this simple interchange, no new additional frequencies are introduced to the attachment. This implies that $T \equiv S$, meaning the initial and the final frequency distributions within the attachment are the same, even though the stiffness of the individual resonators are changed with time in accordance with Eq. (14).

In the spirit of the present context, the system considered here remains conservative even under stiffness modifications. To achieve this goal, the stiffness variation for the $i$ th oscillator would be introduced when $x(t)-x_{i}(t)=0$, such that the perturbation of $\chi_{i}(t)$ does not modify the potential energy stored in the spring, leaving the total energy of the resonator unchanged. Use of this technique suggests the need to introduce the stiffness modifications at different times for each resonator of the set. In practice, however, it is more convenient to modify the stiffness values simultaneously for all the resonators at the same time $t^{*}$ without checking their individual position. In order to make the process simpler, the modified spring stiffness for each oscillator $k_{i}^{\text {new }}$ must have the same energy as the original one (stiffness $k_{i}^{\text {old }}$ ),

$$
\begin{aligned}
& \frac{1}{2} k_{i}^{\text {new }}\left[x\left(t^{*}\right)-x_{i}^{\text {new }}\left(t^{*}\right)\right]^{2}=\frac{1}{2} k_{i}^{\text {old }}\left[x\left(t^{*}\right)-x_{i}^{\text {old }}\left(t^{*}\right)\right]^{2}, \\
& x_{i}^{\text {new }}\left(t^{*}\right)=x\left(t^{*}\right)-\sqrt{\frac{k_{i}^{\text {old }}}{k_{i}^{\text {new }}}}\left[x\left(t^{*}\right)-x_{i}^{\text {old }}\left(t^{*}\right)\right] .
\end{aligned}
$$

Thus, by modifying the stiffness, the corresponding elongations $x_{i}^{\text {new }}\left(t^{*}\right)$ of the spring for each oscillator is also modified with respect to its original values $x_{i}^{\text {old }}\left(t^{*}\right)$, in accordance with the energy conservation requirement expressed above. This condition implies that the energy balance of each oscillator is preserved, but with a different static equilibrium position after the stiffness change.

A final consideration concerns the selection of the time $t^{*}$. This is roughly the time it takes for the energy of the 


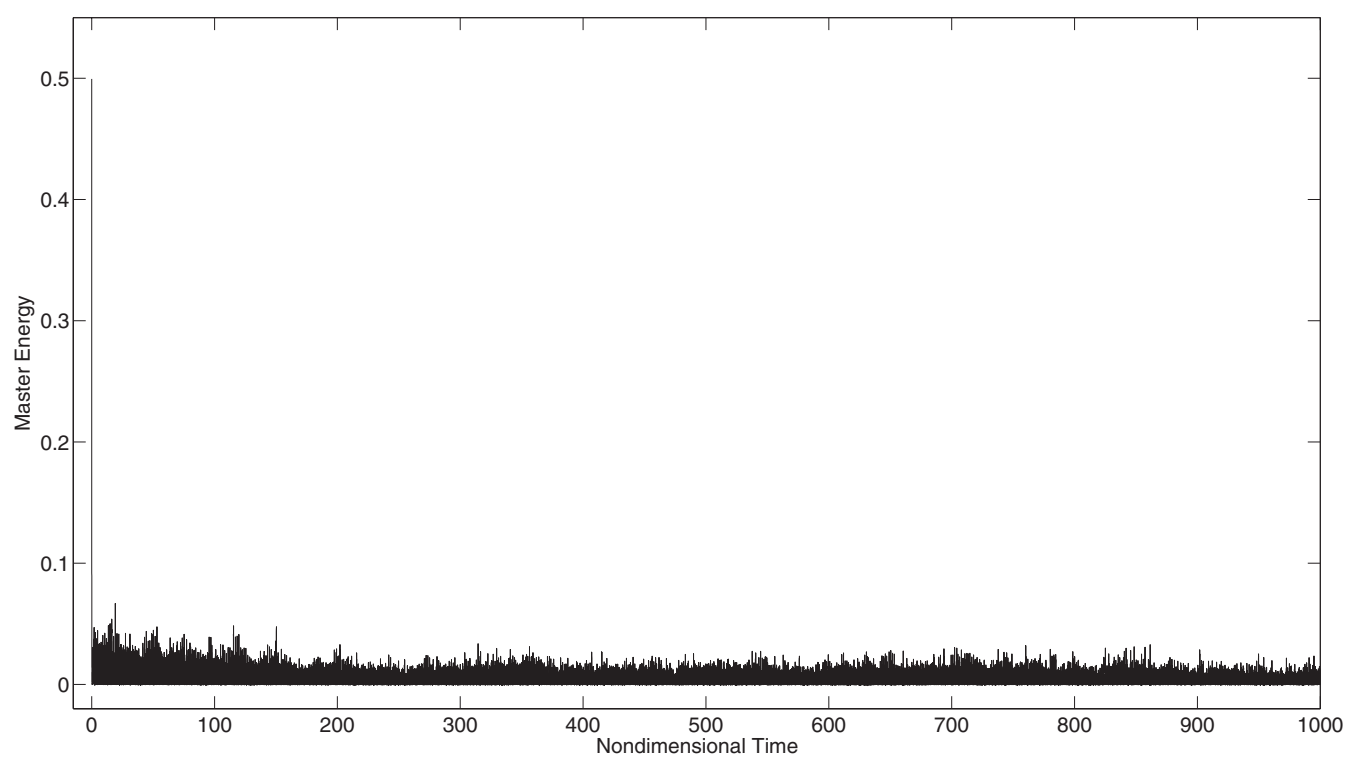

FIG. 4. Energy time history of the master for the nonlinear system.

master to completely migrate to the attachment. As shown in Ref. 7, for a linear attachment, the return time $t_{\text {ret }}$ indicates the time after which the energy returns back to the master, and in Ref. 7 it is also shown how it depends on the selected frequency distribution within the attachment and on the total number $N-1$ of resonators. The time periods of $t_{\text {ret }}$ and $t^{*}$ are similar; in fact, as the energy of the master is transferred to the attachment, phase synchronization among the resonators within the attachment takes place and the energy is suddenly returned to the master.

Therefore, time $t^{*}$ must be long enough to allow the most effective energy transfer from the master to the attachment, but shorter than $t_{\text {ret }}$ to avoid the energy reverse process. A suitable choice for $t^{*}$ could be $t^{*} \approx 0.9 t_{\text {ret }}$ (the one used in the simulations) so that the return effect is prevented and the energy absorbed from the master is nearly all confined in the resonators of the attachment.

\section{NUMERICAL RESULTS}

As a numerical implementation of the model defined in Sec. II, based on elastic collisions, consider a case in which a total of $N=130$ attached oscillators have equally spaced frequencies within the bandwidth $\left[\omega_{M} / 50,2 \omega_{M}\right]$. The mass ratio between the mass of the attachment and the mass of the master is about 0.1 , and $m / M=1 /(10 N)$.

The choice of the characteristic gap $g$ can be operated, following many different criteria. For the present numerical

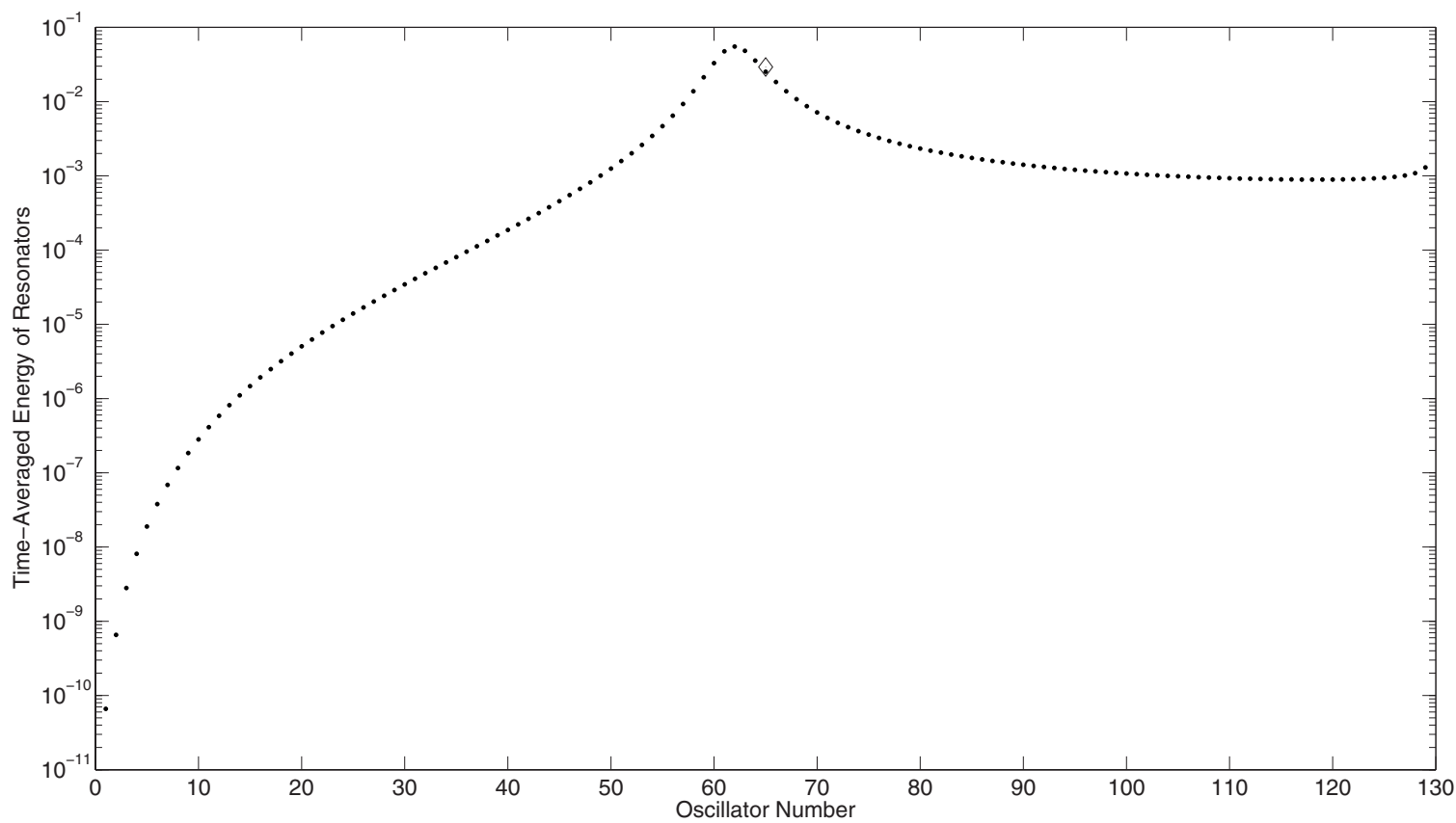

FIG. 5. Time-averaged energy of each resonator for the linear system: dots represent the oscillators energies and the diamond represent the energy of the master; $y$-axis is log-scale. 


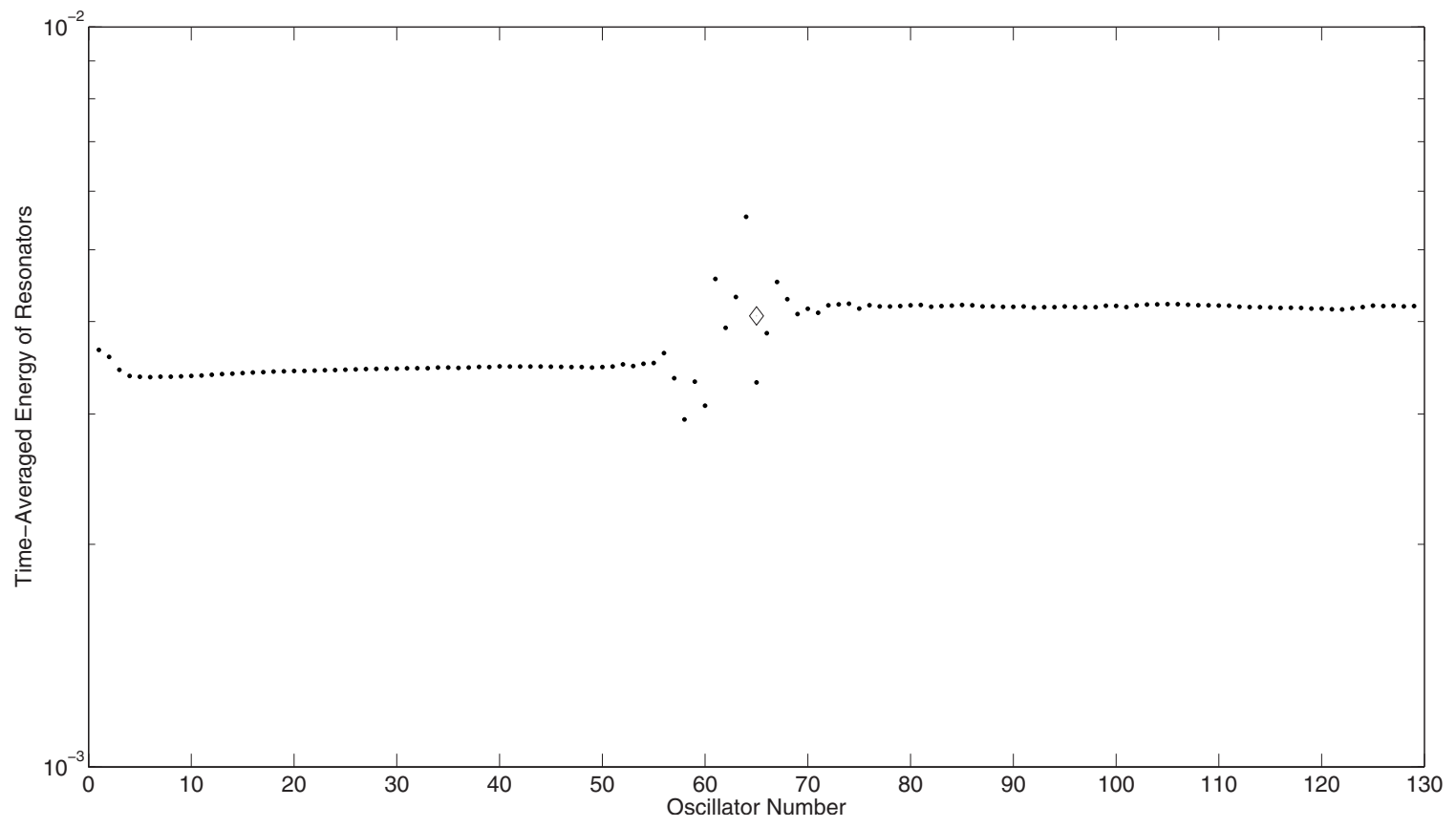

FIG. 6. Time-averaged energy of each resonator for the nonlinear system (symbols as in Fig. 5).

simulations, the steps have been used as follows:

- the system response is simulated, without elastic collisions, during the time interval $\left[0,2 T_{\max }\right]$, where $T_{\max }$ is the maximum natural period of the system;

- the distances $d_{i}(t)=\left|x_{i+1}(t)-x_{i}(t)\right|$, where $i=1, N-2$, between neighbors resonators are monitored, and their maxima $D_{i}$ within the time interval $\left[0,2 T_{\max }\right]$ are extracted; and
- if $g_{\max }=\max \left\{D_{i}, i=1, N-2\right\}$ then the gap $g$ is chosen (equal for all the resonator pairs) as a fraction of $g_{\max }$, namely, $g=0.8 g_{\max }$.

The idea behind this procedure is physically simple: the process of collision is initially activated for those resonators having an energy level close to their maximum. It is empirically found that this criterion produces good results, and a

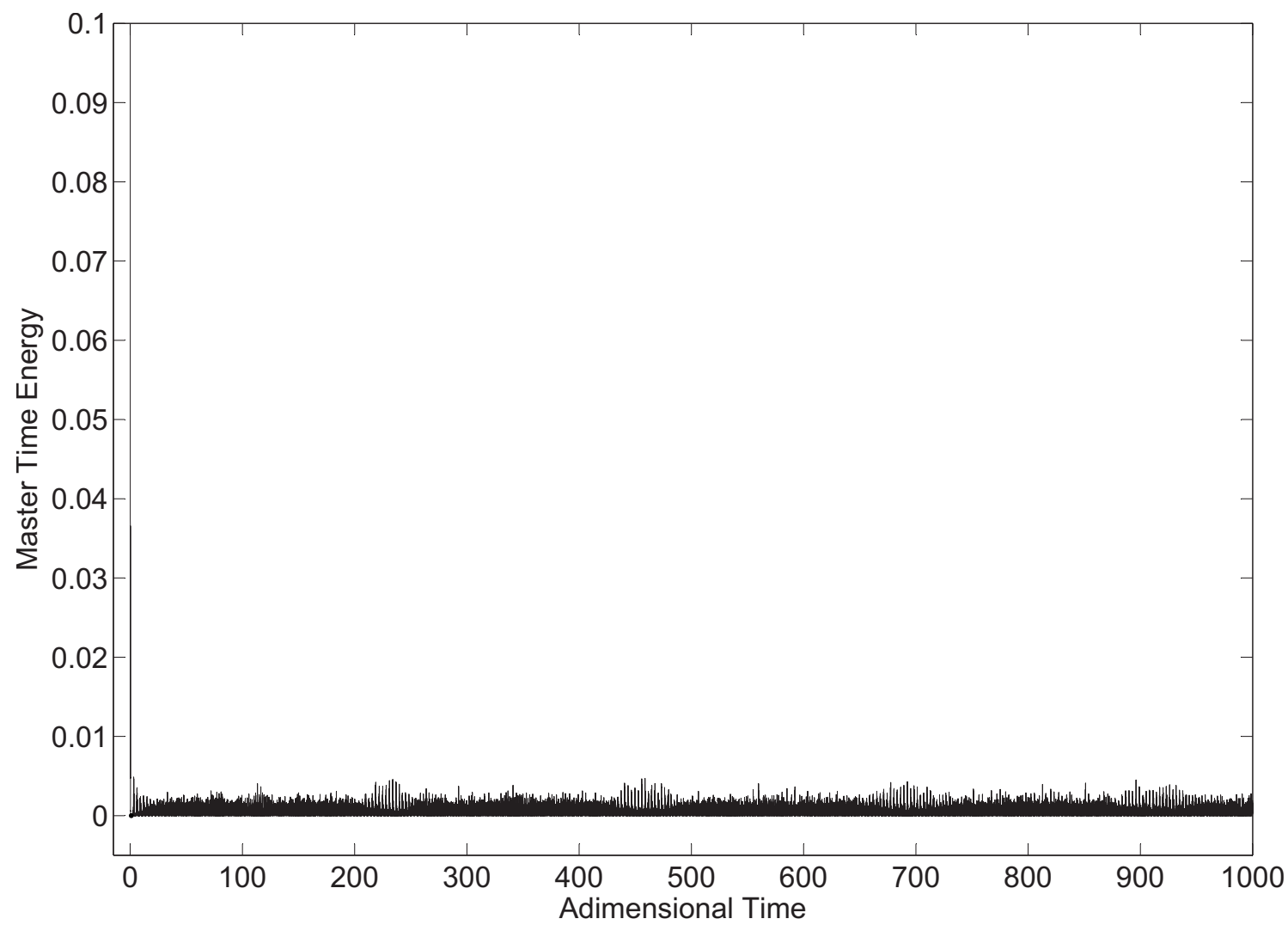

FIG. 7. Energy time history of the master (case a). 


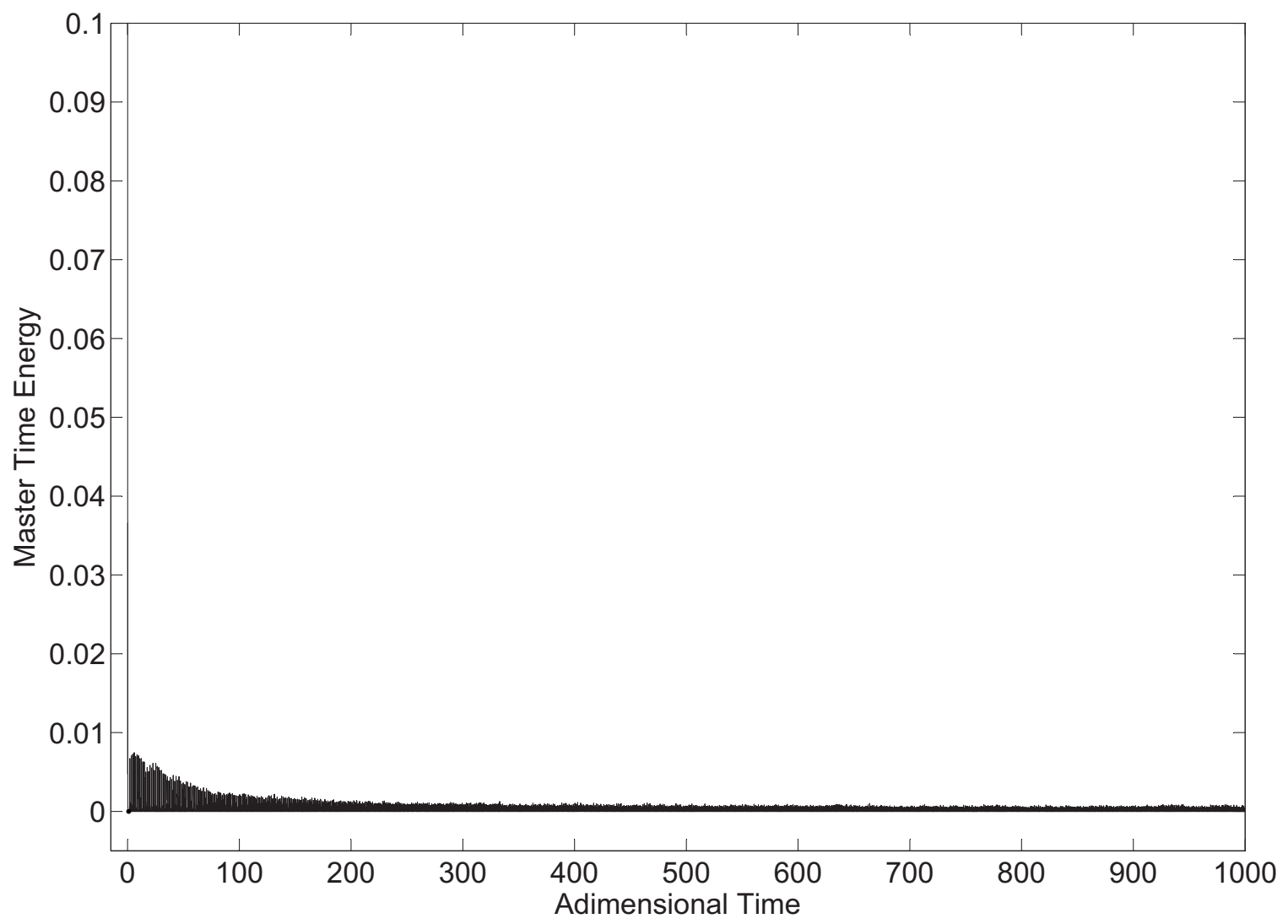

FIG. 8. Energy time history of the master (case b).

systematic analysis of the effect of $g$ on the energy absorption capability of the attachment will be the subject of future investigations.

The total initial energy imparted on the primary is $E_{\text {tot }}$ $=0.5$. Energy time histories of the master are plotted in Figs. 3 and 4 for the linear and nonlinear systems, respectively. Time axis is non-dimensional, taking $T_{\max }$ as the reference time. Presence of impacts enhances the energy absorption capability of the attachment, significantly reducing the vibration amplitude of the master.

Figures 5 and 6 show the time-averaged energy stored in each resonator for the cases of linear and nonlinear systems, respectively. The time base over which the average is computed is equal to $1000^{*} T_{\max }$. For the linear case the energy

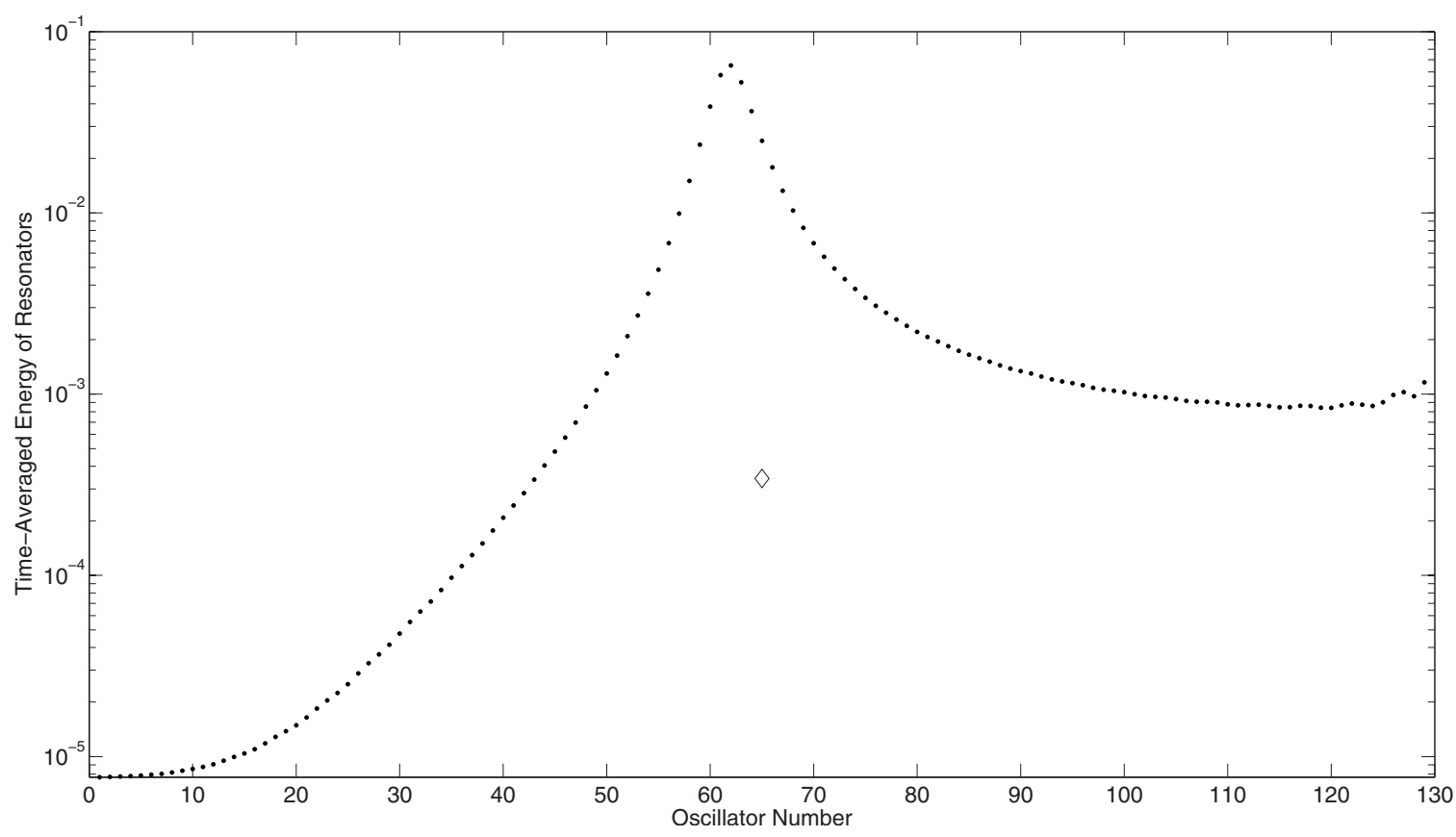

FIG. 9. Time-averaged energy of each resonator (case a) (symbols as in Fig. 5). 


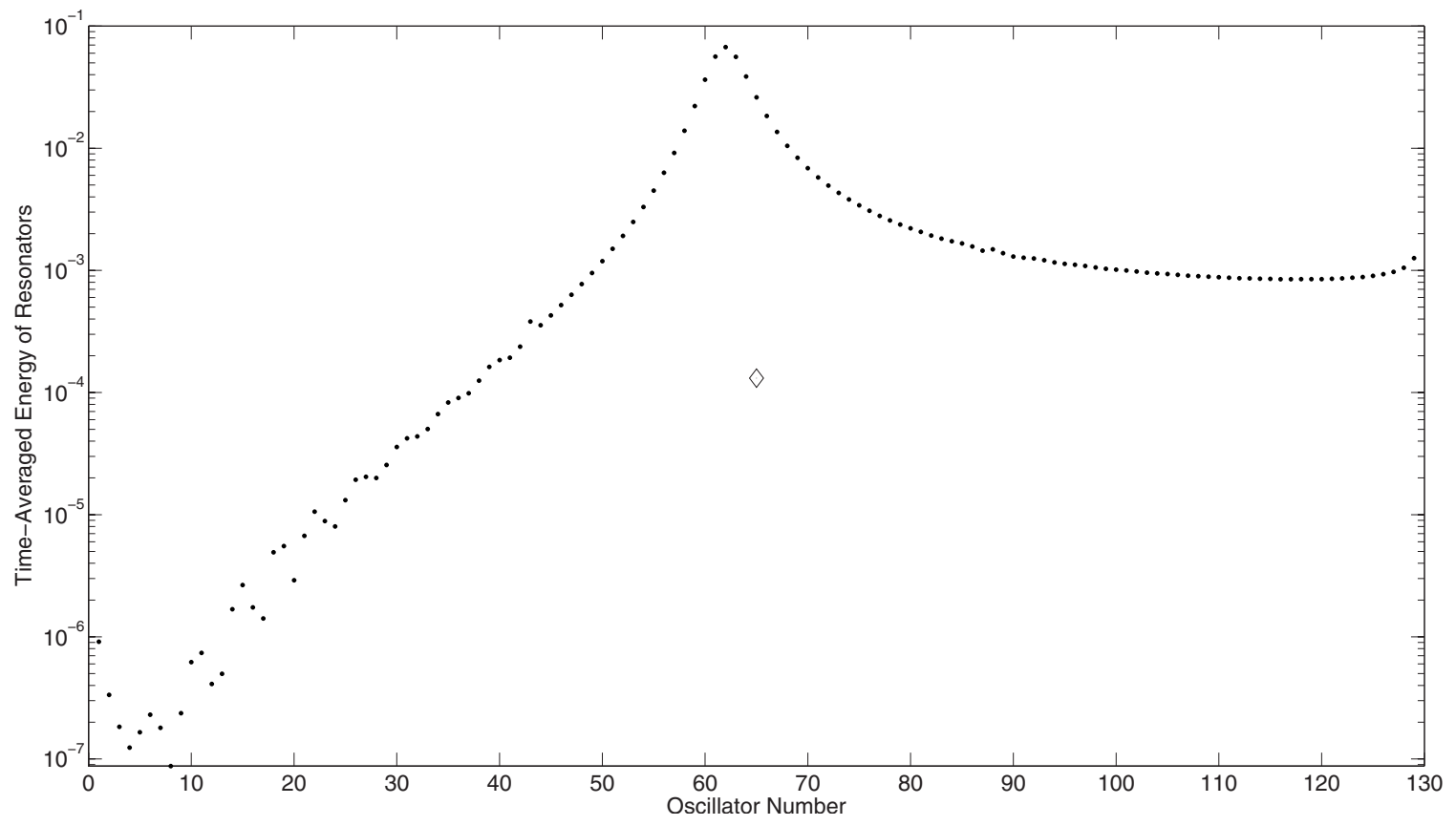

FIG. 10. Time-averaged energy of each resonator (case b) (symbols as in Fig. 5).

is mainly shared among the master and a small group of resonators tuned to the master's frequency, in agreement with the findings of Ref. 7. In fact, in the curve of Fig. 5, a sharp peak appears around the master frequency. In the nonlinear case, energy in the attachment is almost equally shared among resonators, mostly with a value around $E_{\text {tot }} / N$ $\approx 0.0038$, approaching energy equipartitioning.

Turning to the alternative mechanism of introducing nonlinearity through stiffness modification, a system with the same bandwidth and number of degrees of freedom as in the previous case is considered. As shown in Sec. III, the two cases investigated are as follows:

a. parametric control by hardening all the stiffness of the satellite structure, by setting $\Delta k_{i}=k_{N-1}-k_{1}$, such that all the frequencies of the satellites for $t>t^{*}$ fall outside the bandwidth $B$; and

b. parametric control by using the same frequency distribution.

Energy time histories of the master are shown in Figs. 7 and 8 for cases (a) and (b), respectively.

Comparing these results with those in Fig. 3 demonstrates how effective parametric control can be in making the master time energy approach zero.

Figures 9 and 10 show the time-averaged energy stored into each resonator, again for cases (a) and (b), respectively. In both cases the energy of the master is much lower than the equipartition value $E_{\text {tot }} / N \approx 0.0038$, and the first frequency shift starts when the master energy is very close to zero. Comparing Figs. 9 and 10 with Fig. 6, a very limited spreading of the energy among resonators is observed; the shape of the energy spectra shown in Figs. 9 and 10 is much closer to the one obtained for the linear case, as shown in Fig. 5, than to the one with impacts (Fig. 6).
Figures 4, 7, and 8 show that the master energies are quite similar for both cases, as well as impacts and stiffness modification among resonators of the satellite structure, although they are based on different physical phenomena. As shown in Figs. 6, 9, and 10, in the case of impacts, total energy is equally spread among the resonators, thus the energy of the master is close to $E_{\text {tot }} / N$, while in the case of frequency shifts through stiffness modifications there is no equidistribution; the energy is trapped in a group of satellite resonators, and the master energy remains nearly constant and equal to its value at the time of the first shift.

As a final point, energy equipartition within the attachment can be produced by (i) a nonlinear mechanism, through elastic impacts among the resonators and regardless of the frequency distribution of the system, and (ii) a purely linear mechanism from a proper selection of frequency distribution of the oscillators, as recently shown in Ref. 13.

\section{SUMMARY AND CONCLUSIONS}

The present paper considers the problem of energy sharing between a master and a plurality of parallel resonators attached to it, introducing two elements of novelty with respect to the previous investigations regarding the presence of collisions among the resonators within the attachment and parametric variation of their stiffness. Purpose of both of these approaches is to make the energy transfer from the primary to the attachment permanent. For both cases, numerical results show very good energy absorption capability of the attachments introduced in this paper. They may be considered as alternatives to selecting special frequency distributions within the attachment ${ }^{10}$ to produce a nearirreversible energy transfer from the master to an attached set of linear oscillators. Of note, the results obtained with the techniques described here are not significantly sensitive to 
the particular frequency distribution within the attachment. Moreover, the nonlinear mechanisms introduced here produce an energy absorption capability of the attachment that is very similar to that obtained using the previously reported special frequency distribution in linear oscillators. A physical reason that can qualitatively explain this equivalence relies on the ability of the nonlinear mechanisms introduced here in spreading the energy across the resonators of the attachment, an ability that is shared with linear systems having the particular frequency distribution reported in Ref. 10.

${ }^{1}$ A. D. Pierce, V. W. Sparrow, and D. A. Russel, "Fundamental structuralacosutic idealization for structure with fuzzy internals," J. Vibr. Acoust. 117, 339-348 (1995).

${ }^{2}$ M. Strasberg and D. Feit, "Vibration damping of large structures induced by attached small resonant structures," J. Acoust. Soc. Am. 99, 335-344 (1996).

${ }^{3}$ G. Maidanik, "Induced damping by a nearly continuous distribution of a nearly undamped oscillators: Linear analysis," J. Sound Vib. 240, 717-731 (2001).

${ }^{4}$ R. J. Nagem, I. Veljkovic, and G. Sandri, "Vibration damping by a continuous distribution of undamped oscillators," J. Sound Vib. 207, 429-434 (1997).
${ }^{5}$ R. L. Weaver, "The effect of an undamped finite degree of freedom 'fuzzy' substructure: Numerical solution and theoretical discussion," J. Acoust. Soc. Am. 100, 3159-3164 (1996).

${ }^{6} \mathrm{R}$. L. Weaver, "Equipartition and mean square response in large undamped structures," J. Acoust. Soc. Am. 110, 894-903 (2001).

${ }^{7}$ A. Carcaterra and A. Akay, "Transient energy exchange between a primary structure and a set of oscillators: Return time and apparent damping," J. Acoust. Soc. Am. 115, 683-696 (2004).

${ }^{8}$ I. M. Koc, A. Carcaterra, Z. Xu, and A. Akay, "Energy sinks: Vibration absorption by an optimal set of undamped oscillators," J. Acoust. Soc. Am. 118, 3031-3042 (2005).

${ }^{9}$ A. Carcaterra, A. Akay, and I. M. Koç, "Near-irreversibility in a conservative linear structure with singularity points in its modal density," J. Acoust. Soc. Am. 119, 2141-2149 (2006)

${ }^{10}$ A. Carcaterra and A. Akay, "Theoretical foundation of apparent damping and energy irreversible energy exchange in linear conservative dynamical systems," J. Acoust. Soc. Am. 121, 1971-1982 (2007).

${ }^{11}$ A. Akay, Z. Xu, A. Carcaterra, and I. M. Koç "Experiments on vibration absorption using energy sinks," J. Acoust. Soc. Am. 118, 3043-3049 (2005).

${ }^{12}$ L. Zuo and S. A. Nayfeh, "Minimax optimization of multi-degrees of freedom tuned-mass-dampers," J. Sound Vib. 272, 893-908 (2004).

${ }^{13}$ N. Roveri, A. Carcaterra, and A. Akay, "Energy equipartition and frequency distribution in complex attachments," J. Acoust. Soc. Am. 126, 122-128 (2009). 